\title{
Service Restoration in DG-Integrated Distribution Networks Using an Exact Convex OPF Model
}

\author{
H. Sekhavatmanesh, Student Member, IEEE, M. Nick, Student Member, IEEE, M. Paolone, Senior Member, IEEE \\ and R. Cherkaoui, Senior Member, IEEE \\ Distributed Electrical Systems Laboratory (DESL) \\ École Polytechnique Fédérale de Lausanne EPFL \\ Lausanne, Switzerland \\ \{ hossein.sekhavat ; mostafa.nick ; mario.paolone ; rachid.cherkaoui \}@epfl.ch
}

\begin{abstract}
The ever-increasing requirements for reliability and quality of supply suggest to enable the self-healing features of modern distribution networks. Within the context of Active Distribution Networks (ADNs), new, fast and efficient restoration strategies can be developed relying not only on switching devices to realize load transfer operation, but also on the contribution of Distributed Generators (DGs) while controlling their set points. In this paper, a global optimization method is proposed for the restoration problem in a DG-integrated distribution network. The objective is to restore a maximum of loads with a minimum number of switching operations. In order to check the technical constraints of the resulting configuration (ex., line current and bus voltage constraints), a recently published method for exact convex formulation of the OPF problem is incorporated making the restoration problem robust particularly in the case of high nodal injections. The proposed restoration model is formulated as a mixed-integer second-order cone programming (MISOCP) problem. Two test cases are used to quantify the DG effects on the quality of the restoration strategy and to demonstrate the feasibility of the identified solution in cases where general relaxation methods for OPF problem leads to inexact solutions.
\end{abstract}

Index Terms - Active Distribution Network, Convex Relaxation, Distributed Generator, Switching device, optimal power flow, Restoration, Self-healing.

\section{INTRODUCTION}

Following a fault event and its subsequent isolation in a distribution network feeder, customers downstream of the fault location remains unsupplied (Fig. 1). According to the restoration task, the status of certain tie/sectionalizing switches in the network should be changed in the most optimal and rapid way providing other supply paths from the substations to the affected customers. The objective is to restore as many customers as possible with the minimum number of switching operations. It is worth to point out that all the network security constraints (such as voltage and current limits) must be well respected in the new network configuration during all the period where it is operating (restorative period). Therefore, Distribution Network Operators (DNOs) are usually faced with a challenging task in taking the best decision for the restoration service under the pressure of an actual emergency. The need for an operator decision support is becoming more crucial with the ever-increasing load demand and reliability requirements in distribution networks. In order to support DNOs, monitoring, control and communication technologies designed for Smart
Grids should be deployed leading to an autonomous decision making tool for the restoration service known as self-healing mechanism [1].

The restoration process in passive distribution networks counts only on the load transfer from the unsupplied area of the faulted feeder to the neighboring feeders using only switching operation. Today, in Active Distribution Networks (ADNs), new, fast and efficient restoration strategies can be developed incorporating also the set point modifications of Distributed Generators (DGs) in the restoration strategies. In this regard, there are many studies in the literature on DG-aided service restoration. They propose to set up intentional DG-island systems [2] using different methodologies. Each of them supply locally a group of nodes during the restorative period. The authors in $[2,3]$ use a clustering strategy to partition the unsupplied part of the network into self-sufficient DG-island systems. The sizes of the clusters are determined using a rollinghorizon optimization method with the aim of supplying a maximum of loads with minimum operational DG costs. However, these costs are not of interest for the restoration service. The DG-island systems proposed in [4-6] are set up for the restoration service by searching for feasible supply routes in the network graph considering each DG as a possible power source candidate. In these studies, heuristic approaches guided by expert-based ruled are used to find the suitable DG-island system regarding the targeted restoration objectives.

All the above-mentioned studies take into account for different DG operational requirements while setting up those islanding systems. These requirements are, among others, DG black start capability, generation capacity limits, and load pickup capability. However, according to [2], when an intentional DG-island system is planned, other requirements in addition to the ones related to the DG itself must be also considered. These requirements concern among others the protection equipment and settings, voltage regulation equipment, and proper operation of loads in the DG-island system. These requirements are all neglected in the abovementioned studies. Consequently, in the restoration strategy proposed in this paper all the DGs are assumed to be operated in the grid-connected mode only. Thus, the restoration solution will be compatible with respect to all the currently used operation standards. 
The restoration problem is a combinatorial and multidisciplinary problem applying simultaneously knowledge from graph theory and electrical power system discipline. From the mathematical programming point of view, the restoration problem is a mixed-integer and non-linear non-convex optimization problem. Therefore, some studies used metaheuristic methodologies such as Genetic Algorithm (GA), Particle Swarm Optimization (PSO) and Tabu Search to solve such a combinatorial optimization problem (see [8] for a comprehensive technical review). These methods, however, are in general time-consuming and fail to give even a feasible solution in a reasonable time complying with online operation requirements. Therefore, heuristic approaches based on expert systems are proposed for the restoration problem to achieve a near-optimal solution in a short time. In these methods, a set of expert-based rules is used to perform a guided search (not an exhaustive search) inside some sets of possible solutions and choose a suitable one. These approaches use graph search methods suitable for specific network topologies. Actually, they cannot be generalized for any network topology [3], [6-7]. Moreover, in all the published works using these methods, the feasibility of the solution concerning the technical constraints (ex., voltage and current limits) is either ignored [10] or checked at the last stage by a load flow simulation but with a scarce feedback on the modification of the identified restoration solution [9]. Indeed, if the solution is not feasible, it is removed from the search space and the process is repeated to find a new solution. Some papers sort the possible combination of loads that can be restored and check their restoration feasibilities progressively [6]. All these approaches can be very time consuming or lead to a solution very far from the optimal one.

With the ever-growing dimension and complexities of distribution networks, the drawbacks related to the abovementioned strategies are inhibiting their use in actual ADNs. Therefore, in this paper, a convex optimization model is derived for the restoration problem and solved thanks to a rigorous mathematical programming method. The main drawback of the existing literature related to the restoration techniques in ADNs is associated with the appropriate integration of the OPF with regard to its non-convexities. Recently, several works propose to convexify the OPF, e.g., [8-10], using: Semi Definite Programming (SDP) and Second Order Cone Programming (SOCP) relaxations. However, these relaxed formulations of the OPF might lead to technically infeasible solutions due to the inexactness of the optimal solution [14]. It is shown in [13] that using appropriate and more conservative constraints for the lines' ampacity limits and nodal voltage-magnitude limits ensure, under mild conditions, the exactness of the optimal solution. Moreover, the formulation of ampacity constraints needs to properly account for the line transverse parameters. It is more significant in the case of underground co-axial powercable, whose parallel capacitive parameters cannot be neglected. In this respect, we have employed the exact convex OPF model proposed in [13], where the lines' transverse-parameter as well as the network static operational constraints (lines ampacity limits and nodal voltage magnitudes) are appropriately included. This non-trivial coupling results in an exact optimization model

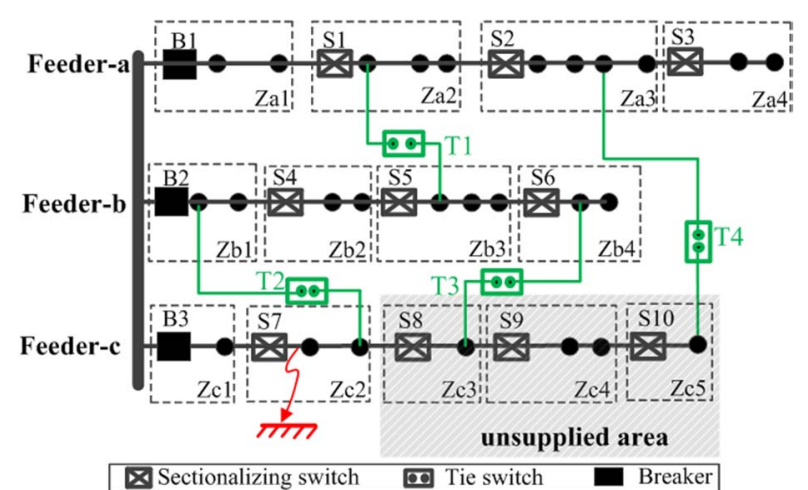

Fig. 1. A Simple schematic of a distribution network under a fault condition

for the restoration service in the form of a MISOCP problem that is the subject of this paper. The dispatchable DGs in the unsupplied area and available feeders (referred as reduced network, hereafter) are incorporated in the restoration process and their optimal set point during the restorative period is derived.

The remainder of this paper is organized as follows. Section II describes the mathematical formulation providing the clustering formulation, then incorporating the proposed clustering strategy into the OPF problem. Section III illustrates two simulation scenarios verifying the main contributions of this paper, which are highlighted in the conclusion given in section IV.

\section{MATHEMATICAL FORMULATION}

In this section, it is aimed to provide a convex analytical optimization model for the restoration problem while accounting for the operational limits using the full constraints of an AC-OPF problem. The problem of computation burden associated with mathematical optimization methods is alleviated in this paper reducing the size of the network that is subject to the optimization problem. Referring to Fig. 1, as an example, this reduced model contains the unsupplied area along with only those healthy feeders that can be directly connected to the unsupplied area through tie-switches (T3 and T4). Those feeders and switches are called available feeders and available tieswitches, respectively. Outside this reduced part of the network nothing is considered to be changed. By doing so, it will be easier for DNO to return the network to its topology in the normal state once the fault is cleared. Thanks to the power flow equations that are incorporated into the optimization problem, further feasibility checking is not anymore required. Therefore, the presented strategy has the advantages of both global optimization and operational research methods resulting in a global optimal solution in a very fast way.

In this paper, a zone is referring to each segment of the feeder that is surrounded by two or more sectionalizing switches. Besides the normally closed and normally open switches shown in Fig. 1, there are breakers at each node for the load (LV network) connection/rejection. The MV network under study is assumed to be balanced and is therefore represented by a directsequence equivalent model. It is also assumed that the fault location has been already diagnosed and isolated. For example, in Fig. 1, the switches s7 and s8 are locked as open to isolate the 
fault. Moreover, the breaker upstream of the faulted feeder (B3 in Fig. 1) has been reconnected to re-supply the upstream area with respect to the fault location (zone Zc1 in Fig. 1). Therefore, the unsupplied area (shaded area in Fig. 1) and the list of available tie-switches (T3 and T4, in Fig. 1) are known. To each of these available tie-switches a group of zones has to be assigned. This group of zones defines a cluster. In this way, the restoration problem is translated into finding the optimal cluster of zones in the unsupplied area to be picked up by each available tie-switch.

The verbally-described problem mentioned above can be translated into a formal optimization problem. The main decision variables in the proposed formulation are the following binary variables:

- $T_{k}=1$ if tie-switch $k$ in the unsupplied area is chosen to be used for the restoration,

- $C_{k, h}=1$ if zone $h$ is included in the cluster associated with tie-switch $k$,

- $L_{i}=1$ if LV network connected at bus $i$ is decided to be restored.

where, $k, h$, and $i$ are indices of tie-switches, zones, and nodes, respectively.

The objective function of the optimization problem is formulated as follows:

$$
\begin{gathered}
\text { Minimize: } F^{o b j}=W_{R} \cdot F^{R e l}+W_{S} \cdot F^{S w}+W_{T} \cdot F^{T e c h} \\
F^{R e l}=\sum_{i \in N^{*}}\left(\left(1-L_{i}\right) \cdot D_{i} \cdot P_{i}\right) \\
F^{S w}=\sum_{(i, j) \in T_{a v a}} T_{i j} \cdot \lambda_{i j}+\sum_{(i, j) \in T_{i n t}} X_{i j} \cdot \lambda_{i j} \\
+\sum_{(i, j) \in S^{*}} S_{i j} \cdot \lambda_{i j}+\sum_{i \in N^{*}} B_{i} \cdot \lambda_{i} \\
F^{\text {Tech }}=\sum_{t} \sum_{(i, j) \in E} F_{i j, t}:\left(F_{i j, t} \geq f_{i j}^{t h r^{2}}\right)
\end{gathered}
$$

Subject to:

\section{Clustering constraints \\ OPF constraints}

where $F^{R e l}, F^{S w}$ and $F^{\text {Tech }}$ are respectively reliability, switching, and technical terms of the objective function while $W_{\mathrm{Re}}, W_{\mathrm{Sw}}$ and $W_{\mathrm{T}}$ are the corresponding weighting factors; $L_{i}$ is a binary decision variable indicating if the load at node $i$ is supplied or rejected $(1 / 0) ; D_{i}$ and $P_{i}$ are respectively the importance factor and the nominal active power consumption of the load at node $i ; \lambda_{i j}$ is the priority factor of tie and sectionalizing switches on line $i j$ and $\lambda_{i}$ is the priority factor of the load breaker at node $i$ (the values of $\lambda_{i j}$ and $\lambda_{i}$ depend on the time needed to operate the corresponding switches); $X_{i j}$ determines if line $i j$ is energized or not; $S_{i j}$ and $B_{i}$ are respectively the indicator $(0 / 1)$ of sectionalizing switch on line $i j$ and load breaker at node $i$ indicating whether it is operated or not; $F_{i j, t}$ is the square of current flow magnitude in line $i j$ at time $t ; f_{i j}^{t h r}$ is the current threshold level at line $i j$ beyond which the current deviation will be minimized; $T_{a v a}$ is the set of available tie-switches; $T_{\text {int }}$ is the set of tie-switches that are totally inside the unsupplied area (internal tie-switches); $S^{*}$ is the set of sectionalizing switches in the unsupplied area; $N$ and $E$ are respectively the set of nodes and lines (incl. tie-lines) in the reduced network while $N^{*}$ and $E^{*}$ are the set of nodes and lines in the unsupplied area.

The reliability objective term expressed in (2) has the most priority accounting for the unrestored loads minimization while considering their priority factors. The second objective function, in terms of priority, is the switching objective which is formulated in (3). This term includes four sub-terms associated, respectively, to the operation of available tie-switches, internal tie-switches, sectionalizing switches, and load breakers at each node in the unsupplied area. The first sub-term is explicitly decided about as a binary variable $T_{i j}$, while the others subterms are function of auxiliary variables $\left(X_{i j}, S_{i j}\right.$ and $\left.B_{i}\right)$ depending on the clustering solution. With the coefficient $\lambda_{i j}$, remotely controlled switches are given a priority over manually controlled ones. The third objective term given in (4) is the operational term minimizing the total deviation of the squared current magnitude beyond a certain threshold $\left(f_{i j}^{t h r}\right)$. The conditional formulation for this squared current flow deviation is linearized according to [15]. For this aim, the auxiliary variables $F_{i j, t}^{*}$ are introduced subject to the following constraints:

$$
\left\{\begin{array}{l}
F_{i j, t}^{*} \geq 0 \\
F_{i j, t}^{*} \geq F_{i j, t}-\left(f_{i j}^{t h r}\right)^{2} \quad \forall(i, j) \in E^{*}, \forall t
\end{array}\right.
$$

Being minimized in (4), the value of auxiliary variables $F_{i j, t}^{*}$ will be bounded to the squared current flow deviation.

\section{A. Clustering Constraints}

The following constraints construct a cluster of surrounding zones for each activated tie-switch $\left(T_{i j}=1\right)$ [21].

$$
\begin{array}{cr}
\sum_{h \in H^{*}} C_{k, h} \leq M \cdot T_{i j} & \forall k:(i, j) \in T_{a v a} \\
T_{i j}+e_{k, h}-1 \leq C_{k, h} & \forall k:(i, j) \in T_{a v a}, h \in H^{*} \\
\sum_{k \in T_{a v a}} C_{k, h} \leq 1 & \forall h \in H^{*} \\
C_{k, i}^{*}=\sum_{h \in H^{*}}\left(C_{k, h} \cdot A_{h, i}\right) & \forall k:(i, j) \in T_{a v a}, i \in N^{*} \\
X_{i}=\sum_{k \in T_{a v a}} C_{k, i}^{*} & \forall i \in N^{*} \\
L_{i} \leq X_{i} & \forall i \in N^{*} \\
C_{k, i}^{*}+C_{k, j}^{*}-1 \leq X_{i j} \leq 1 & \forall(i, j) \in E^{*} \backslash T_{a v a} \\
& \forall k \in T_{a v a} \\
C_{k, i}^{*}-C_{k, j}^{*} \leq 1-X_{i j} \leq 1 & \forall(i, j) \in E^{*} \backslash T_{a v a} \\
C_{k, j}^{*}-C_{k, i}^{*} \leq 1-X_{i j} \leq 1 & \forall k \in T_{a v a}
\end{array}
$$




$$
\begin{aligned}
& 0 \leq X_{i j} \leq \sum_{k \in T_{\text {ava }}}\left(C_{k, i}^{*}+C_{k, j}^{*}\right) \\
& \forall(i, j) \in E^{*} \backslash T_{\text {ava }} \\
& \left\{C_{k, i}^{*}-C_{k, j}^{*} \leq S_{i j} \leq 1\right. \\
& \left\{C_{k, j}^{*}-C_{k, i}^{*} \leq S_{i j} \leq 1\right. \\
& \left(1-L_{i}\right)+X_{i}-1 \leq B_{i} \leq 1 \\
& \forall(i, j) \in S^{*}, k \in T_{\text {ava }} \\
& 0 \leq y_{i j} \leq 1 \quad, \quad 0 \leq y_{j i} \leq 1 \\
& y_{i j}=T_{i j}, \quad Y_{j i}=0 \\
& y_{i j}+y_{j i}=X_{i j} \\
& \sum_{j:(i, j) \in E^{*}} y_{j i}=X_{i} \\
& \forall i \in N^{*} \\
& \forall(i, j) \in E^{*} \\
& \forall(i, j) \in T_{a v a} \\
& \forall(i, j) \in E^{*} \\
& \forall i \in N^{*}
\end{aligned}
$$

where, $X_{i}$ determines if line $i$ is energized or not; $C_{k, i}^{*}$ indicates if node $i$ is included in the cluster associated with tie-switch $k$; $H^{*}$ is set of zones in the unsupplied area; $y_{i j}\left(/ y_{j i}\right)$ is a continuous variable indicating if line $i j$ is oriented from node $i(/ j)$ to node $j(/ i)$ or not $(1 / 0) ; e_{k, h}$ is indicating if zone $h$ is directly connected to tie-switch $k(1 / 0)$; and $A_{h, i}$ is an indicator specifying if node $i$ is in zone $h(1 / 0)$.

Constraint (6) ensures that the cluster corresponding to a nonoperated tie-switch $\left(T_{i j}=0\right)$ should not contain any zone, $M$ being a large multiplier. Constraint (7) verifies that if tie-switch $k$ is operated $\left(T_{i j}=1\right)$, the associated cluster should contain at least zone $h$ that is directly connected to it $\left(e_{k, h}=1\right)$. Constraint (8) states that each zone must be assigned to one cluster at most. The nodes in each cluster are determined by $C_{k, i}^{*}$ in (9) according to the clustering of their hosting zones $\left(A_{h, i}=1\right)$. According to (10), node $i$ is energized if it is included in a cluster $\left(X_{i}=1\right)$. For such an energized node, a decision is made through (11) with a binary variable $L_{i}$ indicating if its load (LV network) will be restored or rejected (1/0). Constraints (12)-(14) identify if line $i j$ that is in the unsupplied area is energized or not $\left(X_{i j}=\right.$ $1 / 0)$. If both ending nodes of line $i j$ are within the same cluster $k$, it is energized (12). Otherwise, if they are in different clusters (13) or within no cluster (14), it means that line $i j$ is not supplied. It should be noted that $X_{i j}$ and $X_{i}$ for line $i j$ and node $i$ that are outside the unsupplied area are equal to one. As given in (3), each internal tie-switch is operated (closed) if the hosting line is energized $\left(X_{i j}=1\right)$. However, the sectionalizing switch on line $i j$ must be operated (opened) only if the ending buses of line $i j$ are assigned to different clusters. This is formulated in (15). According to (16), the load breaker at node $i$ will be opened $\left(B_{i}=1\right)$ only when node $i$ is energized and the same time its loads is decided to be left unsupplied $\left(L_{i}=0\right)$.

Constraints (17)-(20) ensure a radial topology for the unsupplied area based on the directional flow variables $\left(y_{i j}\right.$ and $\left.y_{j i}\right)$ considering graph theory. These constraints are identical to those of [16], except for (20), which is adapted to the proposed clustering approach. According to (18), the flow in the offoutage area can be originated only from one of the activated tieswitches $\left(T_{i j}=1\right)$. Actually, variable $y_{i j}$ indicates the flow orientation in line $i j$ originated from an available tie-switch.

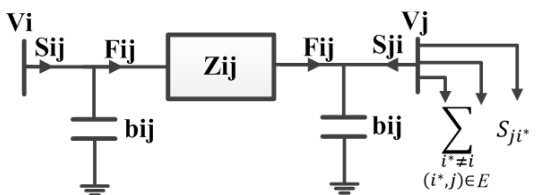

Fig. 2. Classical two-port $\Pi$ model of a distribution cable adopted for the formulation of the OPF relaxed constraints.

Constraint (20) ensures that to each restored node $i\left(X_{i}=1\right)$ exactly one flow must enter guaranteeing the radiality of the network configuration. Although directional flow variables are defined in (17) as continuous variables, they can take only zero or one values as feasible solutions. The proof of this claim is provided in [16].

\section{B. OPF Constraints}

The OPF constraints are included to ensure the feasibility of the solution concerning the technical constraints such as voltage and current limits. In the following, the relaxed formulation of power flow constraints is presented. In order to introduce the part of the nomenclature and for the sake of clarity, the $\Pi$ model of a distribution cable is shown in Fig. 2.

$$
\begin{array}{cr}
0 \leq F_{i j, t} \leq F_{i j}^{\max } \cdot X_{i j} & \forall(i, j) \in E, \forall t \\
0 \leq\left|S_{i j, t}\right| \leq M \cdot X_{i j} & \forall(i, j) \in E, \forall t \\
S_{i, t}=\left(S_{i, t}^{R}-S_{i, t}^{D}\right) \cdot L_{j} & \forall i \in N, \forall t \\
-M .\left(1-X_{i j}\right) \leq V_{i, t}-V_{j, t}-2 \Re\left(z_{i j}^{*}\left(S_{i j, t}+j V_{i, t} b_{i j}\right)\right) \ldots \\
+\left|z_{i j}\right|^{2} F_{i j, t} \leq M \cdot\left(1-X_{i j}\right) & \forall(i, j) \in E, \forall t \\
\sum_{i j, t}=\sum_{\substack{i^{*} \neq i \\
\left(i^{*}, j\right) \in W}} S_{j i^{*}, t}+z_{i j} \cdot F_{i j, t}-j\left(V_{i, t}+V_{j, t}\right) b_{i j}-S_{i, t} \\
\left|S_{i}^{R}\right|_{\max } \geq\left|S_{i, t}^{R}\right| & \forall(i, j) \in E, \forall t \\
F_{i j, t} \geq \frac{\left|S_{i j, t}+j V_{i, t} b_{i j, t}\right|^{2}}{V_{i, t}} & \forall i \in N^{R}, \forall t
\end{array}
$$

where, $\mathfrak{R}$ represents the real part of a complex number; $S_{i}^{D}$ is the complex power demand at node $i ; S_{i}^{R}$ is the complex power of the resource at node $i ; S_{i}$ is the complex power injected at node $i ;\left|S_{i}^{R}\right|_{\max }$ is the apparent power capacity of the resource at node $i ; F_{i j}^{\max }$ is the upper limit of the square of current flow of the line $i j ; M$ is an enough large number; $S_{i j, t}$ is the complex power flow through line $i j$ starting from node $i$ at time $t ; V_{i, t}$ is the square of voltage magnitude at node $i$ and at time $t ; z_{i j}$ is the longitudinal impedance of line $i j ; b_{i j}$ is the susceptance of line $i j$ at each end node; and $N^{R}$ is the set of dispatchable DGs in the reduced network.

Using the first set of constraints (21)-(24), the resulting changes from clustering are mapped on the reduced network to setup completely the corresponding configuration. For this aim, the current flow and active/reactive line flows are forced to be zero for de-energized lines according to (21) and (22). Moreover, the injected active/reactive power for unrestored loads is forced to be zero in (23). In this equation, the resource could be a substation node, an intermittent DG, or a dispatchable DG in the 
studied network. The nodal voltage constraint in [16] is revised in (24) to exclude unrestored lines such as the ones between the clusters. In the second set of constraints, constraint (25) concerns with the active-/reactive power balances at the end buses of each line, excluding load demands at unrestored nodes. According to (26), the apparent power injected by dispatchable DGs in the reduced network is limited to their apparent power capacities. Constraint (27) is the relaxed version of the current flow equation in each line as proposed in [16].

However, it may often occur that the optimal solution of the relaxed OPF, formulated above, does not satisfy the original constraint (i.e. the equality condition in (27)). In particular, this may happen when either one of the nodal voltage upper-bounds or/and line ampacity limits is/are binding. In [13] the authors propose a way to modify the relaxed OPF to ensure the exactness of the relaxation. In particular, they propose to use some auxiliary variables $(\bar{F}, \hat{S}, \bar{S}, \widehat{V})$ to account for the security constraints. $\hat{S}$ and $\hat{v}$ represent the lower bound and upper bound on $S$ and $v$, respectively whereas $\bar{S}$ and $\bar{F}$ are the upper bounds on $S$ and $F$, respectively (see [13] for further details). In the following, the additional constraints are presented concerning the modified relaxed formulation for the OPF problem.

$$
\begin{aligned}
& \hat{S}_{i j, t}=\sum_{\substack{\left.i^{*} \neq i \\
i^{*}, j\right) \in W}} \hat{S}_{j i^{*}, t}-j\left(\hat{V}_{i, t}+\hat{V}_{j, t}\right) b_{i j}-S_{i, t} \\
& \forall(i, j) \in E, \forall t \\
& -M .\left(1-X_{i j}\right) \leq \hat{V}_{i, t}-\hat{V}_{j, t}-2 \Re\left(z_{i j}^{*}\left(\hat{S}_{i j, t}+j \widehat{V}_{i, t} b_{i j}\right)\right) \\
& \leq M .\left(1-X_{i j}\right) \quad \forall(i, j) \in E, \forall t \\
& \bar{S}_{i j, t}=\sum_{\substack{i^{*} \neq i \\
\left(i^{*}, j\right) \in W}} \bar{S}_{j i^{*}, t}+z_{i j} \cdot \bar{F}_{i j, t}-j\left(V_{i, t}+V_{j, t}\right) b_{i j}-S_{i, t} \\
& \bar{F}_{i j, t} V_{j, t} \geq\left|\max \left\{\left|\hat{P}_{j i, t}\right|,\left|\bar{P}_{j i, t}\right|\right\}\right|^{2} \\
& +\left(\max \left\{\left|\hat{Q}_{j i, t}-j \widehat{V}_{j, t} b_{i j}\right|,\left|\bar{Q}_{j i, t}-V_{j, t} b_{i j}\right|\right\}\right)^{2} \\
& \bar{F}_{i j, t} V_{i, t} \geq\left|\max \left\{\left|\hat{P}_{i j, t}\right|,\left|\bar{P}_{i j, t}\right|\right\}\right|^{2} \\
& \begin{array}{r}
+\left(\max \left\{\left|\widehat{Q}_{i j, t}-j \widehat{V}_{i, t} b_{i j}\right|,\left|\bar{Q}_{i j, t}-V_{i, t} b_{i j}\right|\right\}\right)^{2} \\
\forall(i, j) \in E, \forall t
\end{array}
\end{aligned}
$$

where, $P_{i j, t}$ and $Q_{i j, t}$ are active and reactive powers flowing in line $i j$ starting from node $i$, at time $t$.

The grid static security constraints composed by: (i) the nodal voltage magnitudes and (ii) lines ampacity limit constraints, can be formulated as follows (for further details see [13]).

$$
\begin{array}{cr}
V^{\min } \leq V_{i, t} & \forall i \in N, \forall t \\
\widehat{V}_{i, t} \leq V^{\max } & \forall i \in N, \forall t \\
|| \max \left\{\left|\widehat{P}_{j i, t}\right|,\left|\bar{P}_{j i, t}\right|\right\}\left|+j \max \left\{\left|\widehat{Q}_{j i, t}\right|,\left|\bar{Q}_{j i, t}\right|\right\}\right|^{2} \leq V_{j, t} F_{i j}^{\max } \\
|| \max \left\{\left|\widehat{P}_{i j, t}\right|,\left|\bar{P}_{i j, t}\right|\right\}\left|+j \max \left\{\left|\widehat{Q}_{i j, t}\right|,\left|\bar{Q}_{i j, t}\right|\right\}\right|^{2} \begin{array}{l}
\forall(i, j) \in E, \forall t \\
\leq V_{i, t} F_{i j}^{\max } \\
\forall(i, j) \in E, \forall t
\end{array}
\end{array}
$$

where $V^{\max } / V^{\min }$ is the upper/lower limit of the bus voltage magnitudes' square.

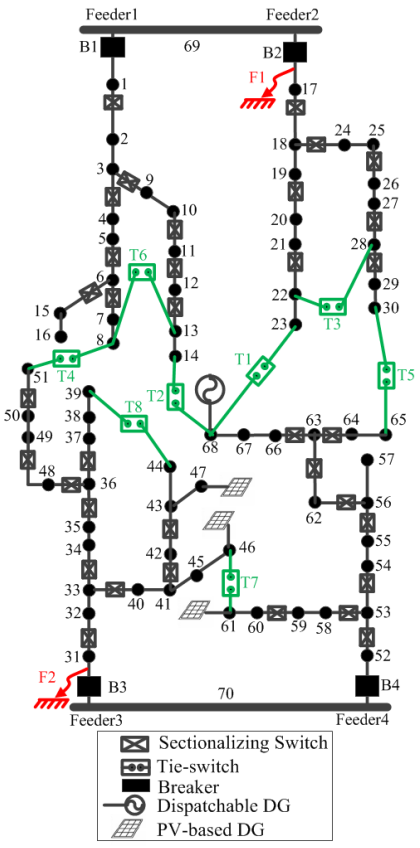

Fig. 3. Test distribution network with 4 feeders and 70 buses [17]

\section{RESULTS AND DISCUSSION}

In order to test the proposed formulation of the restoration problem in ADNs, the $11 \mathrm{kV}$ distribution network introduced in [17] is used. It is shown in Fig. 3. This network includes 2 substations, 4 feeders, 70 nodes, and 76 branches (incl. tiebranches). The system data is given in [17]. The base value for the apparent power is 1 MVA. The current threshold for each line $\left(f_{i j}^{\text {thr }}\right.$ in (4)) is assumed to be $10 \%$ of its current capacity limit. According to ANSI C84.1 standard, the minimum and maximum voltage magnitude limits for the restorative period (as an emergency situation) are relaxed, respectively, to 0.9 and 1.06 p.u. [18]. The majority of nodes along feeders 1-4 in Fig. 3 are assigned, respectively, to industrial, rural, residential, and commercial load types. There is also $10-30 \%$ of public light loads considered at each node. The hourly profile for each of these load patterns is given in [19]. One dispatchable DG with a capacity of $0.6 \mathrm{MW}$ is installed on bus 68 . The set point of this DG is determined by DNO one day ahead, which might be subject to further tuning during the operation (for example, in case of network reconfiguration). The other type of DGs are PVbased DGs installed within LV networks at nodes 46, 47, and 61 with capacities of 0.6, 0.6 and $0.8 \mathrm{MW}$, respectively. The PV generation forecast profiles are obtained from [20]. The restoration algorithm is implemented on a $\mathrm{PC}$ with an $\operatorname{Intel}(\mathrm{R})$ Xeon(R) CPU and 6 GB RAM; and solved in Matlab/Yalmip environment, using Gurobi solver.

Two fault places, one along feeder2 (F1) and one along feeder3 (F2) are considered (see Fig. 3) separately. The restorative period for each simulation scenario is assumed starting from 8:00 A.M. until 10:00 P.M. The voltage set point at each substation node is assumed to be fixed at 1.05 p.u.. Tieswitch T7 and sectionalizing switches on lines 18-24 and 28-29 are remotely-controlled and the rest are all manually-controlled. The priority factors for manually and remotely-controlled 
Table I. Simulation results in case of fault F1

\begin{tabular}{|c|c|c|c|c|c|}
\hline 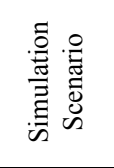 & 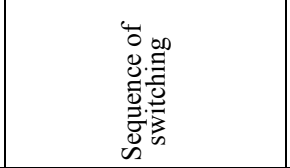 & 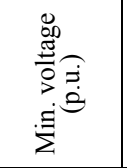 & 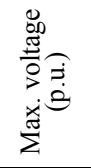 & 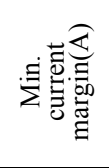 & 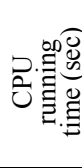 \\
\hline $\begin{array}{c}\text { FaultF1- } \\
\text { DG on } \\
\text { bus } 68 \text { is } \\
\text { not } \\
\text { operated }\end{array}$ & \begin{tabular}{|} 
Open switches \\
$18-24,25-26$, and $28-29$ \\
Open load breakers at \\
buses $18,20,21,28$ \\
Close T3 \\
Close T1 and T5
\end{tabular} & $\begin{array}{c}0.900 \text { p.u. } \\
\text { at node } \\
26 \text { at } \\
\mathrm{t}=18: 00\end{array}$ & $\begin{array}{l}1.050 \\
\text { p.u. at } \\
\text { nodes } \\
69,70 \\
\text { all the } \\
\text { times }\end{array}$ & $\begin{array}{c}11.082 \\
\mathrm{~A} \text { in line } \\
56-62 \text { at } \\
\mathrm{t}=18: 00\end{array}$ & 80 \\
\hline $\begin{array}{l}\text { FaultF1- } \\
\text { DG on } \\
\text { bus } 68 \text { is } \\
\text { operated }\end{array}$ & \begin{tabular}{|c|} 
Open switches \\
$18-24$, and \\
$28-29$ \\
Open load breaker at bus \\
21 \\
Close T3 \\
Close T1 and T5 \\
\end{tabular} & $\begin{array}{c}0.902 \text { p.u. } \\
\text { at node } \\
24 \text { at } \\
\mathrm{t}=18: 00\end{array}$ & $\begin{array}{l}1.050 \\
\text { p.u. at } \\
\text { nodes } \\
69,70 \\
\text { all the } \\
\text { times }\end{array}$ & $\begin{array}{l}15.509 \mathrm{~A} \\
\text { in line } \\
56-62 \text { at } \\
\mathrm{t}=18: 00\end{array}$ & 3.52 \\
\hline
\end{tabular}

switches/load breakers are assumed equal to 1 and 0.05 , respectively. The importance factor of load at buses 19 and 26 is 10 and for all the other nodes is 1.

When fault F1 occurs, once breaker B2 is opened, the DG on node 68 must be disconnected within 2 seconds [2]. In the first simulation scenario, it is assumed that this DG will not be operated anymore during the restorative period. The optimal switching sequence for the restoration strategy along with the unsupplied loads and processing time are shown in Table I.

Regarding the obtained restoration solution, voltage and current profiles are derived with the original model of the network (no reduction) using power flow simulations in Matlab/MATPOWER toolbox. A brief report of these profiles is also shown in Table I. According to the results of the simulation scenario where DG is operated, among other switching actions, switches 18-24 and 25-26 should be opened. It means that the area including nodes 24 and 25 is isolated from the restored areas. This type of manoeuver is missing in the restoration strategies proposed in the literature. Actually, those strategies instead of isolating nodes 24 and 25 by opening the switch 1824 only, would propose to open the load breakers at nodes 24 and 25 , increasing uselessly the number of required switching operation. According to the results shown in Table I for the first simulation scenario, beside the isolated area, the loads at nodes $18,20,21$ and 28 are also unrestored by opening the corresponding breakers. In this case, instead of load 28, loads 19 and 26 could have been rejected, leading to a smaller value of total energy not supplied. However, regarding the higher importance factor of loads 19 and 26, they were preferred to be restored.

In the second simulation scenario, the dispatchable DG at node 68 is assumed to be reconnected at the last stage of switching sequences. The schedule of active/reactive power injection of this DG during the restorative period is determined such that the restoration objectives can be better fulfilled while not exceeding the DG's power capacity. As it can be seen in Table I, the area that was isolated in the first scenario is now supplied and the load at nodes 18, 20 and 28 that were rejected are now restored in this scenario.
Table II. Simulation results in case of fault F2

\begin{tabular}{|c|c|c|c|c|c|}
\hline 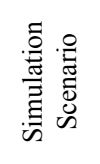 & 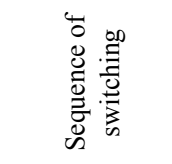 & 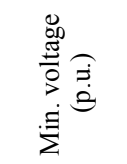 & 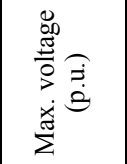 & 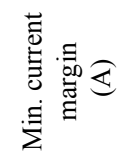 & 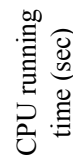 \\
\hline $\begin{array}{c}\text { FaultF2 } \\
- \\
\text { Inexact } \\
\text { OPF }\end{array}$ & $\begin{array}{c}\text { Open switch } \\
50-51 \\
\text { Close T4 and } \\
\text { T7 }\end{array}$ & $\begin{array}{l}0.940 \text { p.u. } \\
\text { at node } 50 \\
\text { at } \mathrm{t}=20: 00\end{array}$ & $\begin{array}{l}1.068 \text { p.u. } \\
\text { at node } 47 \\
\text { at } t=13: 00\end{array}$ & $\begin{array}{l}17.75 \mathrm{~A} \text { in } \\
\text { line } 53-58 \\
\text { at } \mathrm{t}=20: 00\end{array}$ & 11.6 \\
\hline $\begin{array}{c}\text { FaultF2 } \\
- \\
\text { Exact } \\
\text { OPF }\end{array}$ & $\begin{array}{c}\text { Open load } \\
\text { breaker at bus } \\
46 \\
\text { Close T7 }\end{array}$ & $\begin{array}{l}0.924 \text { p.u. } \\
\text { at node } 51 \\
\text { at } \mathrm{t}=18: 00\end{array}$ & $\begin{array}{l}1.050 \text { p.u. } \\
\text { at nodes } \\
69,70 \text { all } \\
\text { the times }\end{array}$ & $\begin{array}{c}3.5 \mathrm{~A} \text { in } \\
\text { line } 53-58 \\
\text { at } \mathrm{t}=20: 00\end{array}$ & 31.7 \\
\hline
\end{tabular}

In case of fault F2, switch 31-32 and breaker B3 are left open to isolate the fault. If the general relaxation method proposed for OPF constraints in [16] is applied, the solution is to restore the whole unsupplied area by opening the switch on line 50-51 and close tie-switches T4 and T7 (see Table II). However, as shown in Table II, this solution is infeasible regarding the technical constraints (upper voltage limit violation). The voltage and current profiles in the network at $\mathrm{t}=1: 00$ P.M., that are derived from the optimization problem are shown in Fig. 4. In order to verify the exactness of this solution, the voltage and current profiles in the new network configuration are determined using a posteriori power flow simulation and the results are shown also in Fig. 4. As it can be seen, the line currents and voltage magnitudes resulting from the optimization problem become inexact in the unsupplied area and Feeder-4 that is mainly restoring the unsupplied area. The reason is that, the reverse power flow injected by intermittent DGs at nodes 46,47 , and 61 causes the upper voltage limit to be binding and, consequently, leading to an inexact solution.

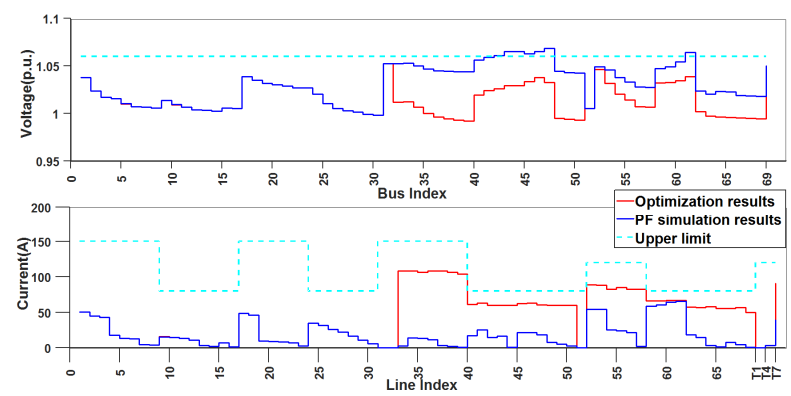

Fig. 4. The voltage and current profiles at $\mathrm{t}=13: 00$ P.M. in the new network configuration derived from the general relaxed OPF formulation and the power flow simulation.

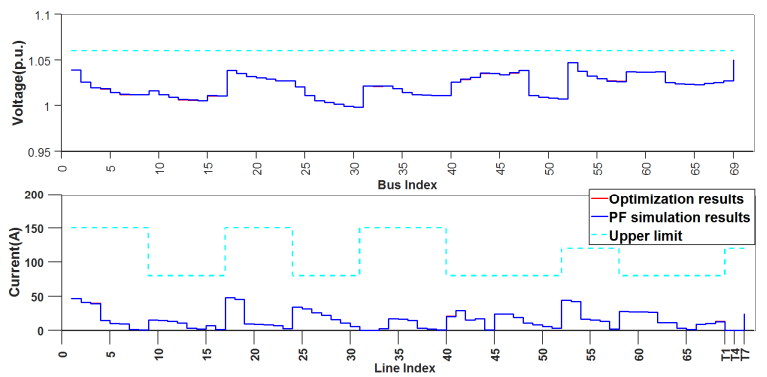

Fig. 5. The voltage and current profiles at $t=13: 00$ P.M. in the new network configuration derived from the modified OPF formulation and the power flow simulation 
Now, if the modified OPF relaxation method is applied, the results is according to the last row of Table II. As it can be seen, the LV network at node 49 (including the connected DG) is rejected to limit the reverse power flow at the substation node. As validated by a posteriori power flow simulation reported in Table II, this solution is feasible regarding the technical constraints (voltage and current limits) all over the network during the restorative period. The voltage and current profiles at $t=1: 00$ P.M. (when the voltage violates the upper limit with the inexact restoration solution) using the modified OPF formulation and the power flow simulation are shown in Fig. 5. As it can be seen, the profiles are matching the exact results derived from the power flow simulation.

\section{CONCLUSION}

This paper presents a MISOCP optimization model for the restoration problem in a DG-integrated distribution network. Exact modeling of the network reconfiguration along with the DG operation for the restoration strategy is the first original contribution of this paper. Considering the network reconfiguration, the proposed formulation is able to represent partial restoration solutions accurately. In such a case, some parts of the unsupplied area are left unrestored to respect the technical constraints in the restored part of the network. Regarding the DG integration in the restoration problem, this paper proposes to operate DG grid-connected mode helping to increase the restored loads or to decrease the number of required switching actions.

In order to check the technical constraints of the network (ex., line current and bus voltage constraints), the power flow equations are included resulting in a nonlinear and nonconvex restoration optimization problem. Recently, relaxation methods have been proposed in the literature for radial distribution networks to convert the AC optimal power flow problem into a convex optimization problem [16]. However, as shown in [13], these methods fail to be exact in the case of networks with high level of DG penetration, which will be the case in future smart grids. Therefore, the second contribution of this paper is to integrate the modified OPF relaxation method in the restoration problem. With this modified formulation, the proposed restoration problem is robust in the case of high nodal injections in the distribution network. In order to illustrate these contributions, the proposed restoration model is successfully tested on a 70-bus distribution network in the case of two fault scenarios. As shown in the previous section, the switching and DG operations are incorporated in the restoration strategy leading to an optimal and feasible solution.

\section{ACKNOWLEDGMENT}

The authors gratefully acknowledge the financial support of the Qatar Environment and Energy Research Institute (QEERI).

\section{REFERENCES}

[1] N. Hatziargyriou, "Distribution systems and dispersed generation," Water Energy Int., vol. 71, no. 1, pp. 37-46, 2014.
[2] T. S. Basso and R. DeBlasio, "IEEE 1547 series of standards: interconnection issues," IEEE Trans. Power Electron., vol. 19, no. 5, pp. 1159-1162, Sep. 2004.

[3] Z. Wang and J. Wang, "Self-Healing Resilient Distribution Systems Based on Sectionalization Into Microgrids," IEEE Trans. Power Syst., vol. 30, no. 6, pp. 3139-3149, Nov. 2015.

[4] S. A. Arefifar, Y. A. R. I. Mohamed, and T. H. M. El-Fouly, "Supply-Adequacy-Based Optimal Construction of Microgrids in Smart Distribution Systems," IEEE Trans. Smart Grid, vol. 3, no. 3, pp. 1491-1502, Sep. 2012.

[5] I. K. Song, W. W. Jung, J. Y. Kim, S. Y. Yun, J. H. Choi, and S. J. Ahn, "Operation Schemes of Smart Distribution Networks With Distributed Energy Resources for Loss Reduction and Service Restoration," IEEE Trans. Smart Grid, vol. 4, no. 1, pp. 367-374, Mar. 2013.

[6] T. T. H. Pham, Y. Besanger, and N. Hadjsaid, "New Challenges in Power System Restoration With Large Scale of Dispersed Generation Insertion," IEEE Trans. Power Syst., vol. 24, no. 1, pp. 398-406, Feb. 2009.

[7] Y. Xu, C. C. Liu, K. Schneider, F. Tuffner, and D. Ton, "Microgrids for Service Restoration to Critical Load in a Resilient Distribution System," IEEE Trans. Smart Grid, vol. PP, no. 99, pp. 1-1, 2016.

[8] Y. Liu, R. Fan, and V. Terzija, "Power system restoration: a literature review from 2006 to 2016," J. Mod. Power Syst. Clean Energy, vol. 4, no. 3, pp. 332-341, Jul. 2016.

[9] J. B. Leite and J. R. S. Mantovani, "Development of a SelfHealing Strategy With Multiagent Systems for Distribution Networks," IEEE Trans. Smart Grid, vol. PP, no. 99, pp. 1-9, 2016.

[10] Y. Xu and W. Liu, "Novel Multiagent Based Load Restoration Algorithm for Microgrids," IEEE Trans. Smart Grid, vol. 2, no. 1, pp. 152-161, Mar. 2011.

[11] L. Gan, N. Li, U. Topcu, and S. Low, "On the exactness of convex relaxation for optimal power flow in tree networks," in 2012 IEEE 51st IEEE Conference on Decision and Control (CDC), 2012, pp. 465-471.

[12] L. Gan, N. Li, U. Topcu, and S. H. Low, "Exact Convex Relaxation of Optimal Power Flow in Radial Networks," IEEE Trans. Autom. Control, vol. 60, no. 1, pp. 72-87, Jan. 2015.

[13] M. Nick, R. Cherkaoui, J.-Y. L. Boudec, and M. Paolone, “An Exact Convex Formulation of Optimal Power Flow in Radial Distribution Networks Including Transverse Components," ArXiv160501964 Math, May 2016.

[14] K. Christakou, D.-C. Tomozei, J.-Y. Le Boudec, and M. Paolone, "AC OPF in radial distribution networks - Part I: On the limits of the branch flow convexification and the alternating direction method of multipliers," Electr. Power Syst. Res., vol. 143, no. Supplement C, pp. 438-450, Feb. 2017.

[15] H. Sekhavatmanesh and R. Cherkaoui, "Optimal Infrastructure Planning of Active Distribution Networks Complying with Service Restoration Requirements," IEEE Trans. Smart Grid, vol. PP, no. 99, pp. 1-1, 2017.

[16] J. A. Taylor and F. S. Hover, "Convex Models of Distribution System Reconfiguration," IEEE Trans. Power Syst., vol. 27, no. 3, pp. 1407-1413, Aug. 2012.

[17] D. Das, "Reconfiguration of distribution system using fuzzy multiobjective approach," Int. J. Electr. Power Energy Syst., vol. 28, no. 5, pp. 331-338, Jun. 2006.

[18] 'Std, A. N. S. I. 'C84. 1-2011.' American National Standard for Electric Power Systems and Equipment-Voltage Ratings (60 Hertz) (2011)."

[19] E. Lopez, H. Opazo, L. Garcia, and P. Bastard, "Online reconfiguration considering variability demand: applications to real networks," IEEE Trans. Power Syst., vol. 19, no. 1, pp. 549553, Feb. 2004.

[20] [Online], "Solar Irradiation Data (SODA)," http://www.sodais.com/eng/index.html. .

[21] H. Sekhavatmanesh and R. Cherkaoui, "Distribution Network Restoration in a Multi-Agent Framework Using a Convex OPF model," IEEE Trans. Smart Grid, vol. PP, no. 99, pp. 1-1, 2018. 